\title{
CEACAM6 is associated with osteosarcoma metastasis and facilitates epithelial-mesenchymal transition in osteosarcoma cells
}

This article was published in the following Dove Press journal:

OncoTargets and Therapy

\author{
Zeyu Wang' \\ Chong Luo' \\ Huidan Wang ${ }^{2}$ \\ Xia Yan ${ }^{2}$ \\ Wei Liu' \\ Zengdong Meng' \\ 'Department of Orthopaedics, The \\ First People's Hospital of Yunnan \\ Province, Dali 650000, Yunnan, \\ People's Republic of China; ${ }^{2}$ Medical \\ Faculty, Kunming University of Science \\ and Technology, Dali 650000, Yunnan, \\ People's Republic of China
}

Correspondence: Wei Liu;

Zengdong Meng

Department of Orthopaedics, The First People's Hospital of Yunnan Province, Dali 650000, Yunnan, People's Republic of China

Tel+86087|6363307|

Email khyygklw@hotmail.com;

menggu7119@vip.sina.com
Background: Carcinoembryonic antigen-related cell adhesion molecule 6 (CEACAM6) is a member of CEACAM family and has been reported to be upregulated in various types of human cancer and involved in tumor progression and metastasis. However, the biological roles and clinical significances of CEACAM6 in osteosarcoma still remain to be elucidated.

Materials and methods: Real-timePCR, immunohistochemistry and Western blot analysis were used to determine CEACAM6 expression in osteosarcoma cell lines and clinical specimens. Then the clinical relevance of CEACAM6 was analyzed in osteosarcoma. The function of CEACAM6 in osteosarcoma was examined by wound-healing and cell invasion assays, and expression levels of epithelial-mesenchymal transition markers.

Results: In the present study, we found that CEACAM6 was markedly upregulated in metastatic osteosarcoma tissues when compared with the nonmetastatic osteosarcoma tissues. Upregulation of CEACAM6 was significantly associated with lung metastasis status $(P=0.006)$ in patients with osteosarcoma. Survival analyses suggested that osteosarcoma patients with high CEACAM6 expression had a significantly shorter overall survival time and lung metastasis-free survival time than those with low CEACAM6 expression. Knockdown of CEACAM6 inhibits osteosarcoma cell migration and invasion. Moreover, silencing CEACAM6 suppressed osteosarcoma cells epithelial-mesenchymal transition.

Conclusion: Taken together, this study suggests that CEACAM6 might be a promising biomarker and a potential therapeutic target for the treatment of metastatic osteosarcoma.

Keywords: CEACAM6, osteosarcoma, metastasis, epithelial-mesenchymal transition, prognosis

\section{Introduction}

Osteosarcoma is the most common type of malignant tumor arising from bone, especially in children and adolescents, with an incidence of 4.4 per million in the USA. ${ }^{1}$ Over the past 30 years, outcomes and treatment for osteosarcoma remain nearly constant. Children and young adults with localized, nonmetastatic disease have a $60 \%-70 \%$ chance of survival 5 years after diagnosis; however, outcomes for patients with metastatic disease are very poor, with a 5-year overall survival (OS) of $23 \%{ }^{2-4}$ Unfortunately, metastases are found in statistically up to $23 \%$ of osteosarcoma patients, and the lung is the most common site. ${ }^{5,6}$ Thus, there is an urgent need for a better understanding of the molecular mechanisms that contribute to the process of osteosarcoma metastasis and for a promising diagnostic and prognostic biomarkers for metastatic osteosarcoma. 
Carcinoembryonic antigen-related cell adhesion molecule 6 (CEACAM6) is a member of the immunoglobulin supergene family. ${ }^{7}$ Previous studies have revealed that CEACAM6 is involved in many crucial cellular events such as cell adhesion, migration, invasion and tumorigenicity. ${ }^{8-10}$ Overexpression of CEACAM6 has been observed in various types of human cancer, including colorectal cancer, breast cancer, ovarian neoplasms and intrahepatic cholangiocarcinoma, and may be associated with tumor progression and metastasis. ${ }^{11-14}$ Recently, Liu et al found that CEACAM6 was notably highly expressed in metastatic osteosarcoma tissues when compared with nonmetastatic osteosarcoma tissues. ${ }^{15}$ However, the mechanisms and clinical significances underlying the participation of CEACAM6 in osteosarcoma metastasis still remain to be elucidated.

In the current work, we confirmed that the expression of CEACAM6 was increased in metastatic osteosarcoma tissues. Overexpression of CEACAM6 positively correlated with lung metastasis status, and predicted poorer prognosis in osteosarcoma. Moreover, silencing CEACAM6 effectively attenuated osteosarcoma cell migration and invasion. In addition, we found that CEACAM6 facilitated epithelialmesenchymal transition (EMT) in osteosarcoma cell lines. Therefore, our research indicates that CEACAM6 plays a pivotal role in osteosarcoma progression and might serve as a promising prognostic biomarker for osteosarcoma.

\section{Materials and methods}

\section{Cell lines}

Human hFOB1.19 osteoblasts, fibroblast NIH3T3 cell lines, and osteosarcoma cell lines, including MG-63, HOS, U-2 OS, Saos-2 and 143B cells, purchased commercially from American Type Culture Collection (ATCC) (Manassas, VA, USA), were used and cultured in DMEM (Invitrogen, Carlsbad, CA, USA) supplemented with $10 \%$ fetal bovine serum (FBS; HyClone, Logan, UT, USA).

\section{Patient information and tissue specimens}

A total of 91 paraffin-embedded osteosarcoma tissues, which had been histopathologically and clinically diagnosed, were collected for this study. Fresh osteosarcoma samples, including three nonmetastatic (primary) and three metastatic osteosarcoma tissues, and two adjacent non-tumor tissues, were obtained from patients who were diagnosed with osteosarcoma. All samples were collected from the First People's Hospital of Yunnan Province. Written informed consent forms to use these tissue samples for research purposes were obtained from all patients. This study was approved by the Ethics Committee of the First People's Hospital of Yunnan Province according to the 1975 Declaration of Helsinki.

\section{Total RNA extraction, reverse transcription (RT) and real-time polymerase chain reaction (PCR)}

Total RNA from fresh tissues was isolated using the Trizol reagent (Invitrogen) according to instructions from the manufacturer, and the mRNA levels of CEACAM6 were evaluated by RT-PCR. First, $2 \mu \mathrm{g}$ of total RNA was reverse transcribed to cDNA using M-MLV Reverse Transcriptase (Promega, Madison, WI, USA) according to the manufacturer's protocol. RT-PCR analysis was performed on an ABI Prism 7500 Sequence Detection System (Applied Biosystems; Thermo Fisher Scientific, Waltham, MA, USA). The results were normalized to the expression of the housekeeping gene GAPDH. Relative expression levels were calculated as $2^{- \text {([Ct of gene] - [Ct of GAPDH]) }}$, in which $\mathrm{Ct}$ represents the threshold cycle for each transcript. The oligonucleotide primers are listed as follows: CEACAM6 (forward, 5'-ACGTCACCCAGAATGACACA-3'; reverse, 5'-GACCATTTACCCACCACAGG-3'); GAPDH (forward, 5'-GTCTCCTCTGACTTCAACAGCG-3'; reverse, 5'-ACCACCCTGTTGCTGTAGCCAA-3').

\section{Plasmids, virus constructs and retroviral infection of target cells}

Human CEACAM6 shRNA clone (HSH011682-CU6) was purchased from GeneCopoeia (Guangzhou, China). The plasmid transfections were performed using the Lipofectamine 3000 reagent (Invitrogen) according to instructions from the manufacturer.

\section{Western blot analysis}

Western blot analysis was performed as previously described, ${ }^{16}$ using antibodies against CEACAM6 (ab154614, 1:200; Abcam, Cambridge, UK), E-cadherin (\#3195, 1:1,000; Cell Signaling, Danvers, MA, USA), N-cadherin (\#4061, 1:1,000; Cell Signaling), vimentin (\#5741, 1:1,000; Cell Signaling), and $\alpha$-tubulin (T6199, 1:2,000; SigmaAldrich, St Louis, MO, USA). $\alpha$-Tubulin was used as the loading control.

\section{Immunohistochemistry}

Immunohistochemical (IHC) analysis was conducted as described previously. ${ }^{17}$ After deparaffinization, the sections were immunohistochemically stained using anti-CEACAM6 (ab154614, 1:200; Abcam). 


\section{Evaluation of immunohistochemistry}

The degree of immunostaining of formalin-fixed, paraffinembedded sections was examined and scored independently by two pathologists. The IHC staining score was determined as previously described. ${ }^{18} \mathrm{~A}$ tumor cell proportion was scored as: 0 , no positive tumor cells; $1,1 \%-25 \%$ positive tumor cells; $2,25 \%-50 \%$ positive cells; $3,50 \%-75 \%$ positive tumor cells and $4,>75 \%$ positive tumor cells. Staining intensity was scored as: 0 , negative; 1 , weak; 2 , moderate and 3 , strong. The staining index (SI) was calculated as the staining intensity score $\times$ the proportion of positive tumor cells (ranging from 0 to 12$)$ and grouped as low $(\mathrm{SI}<6)$ and high $(\mathrm{SI} \geq 6)$ expression.

\section{Wound-healing assay}

MG-63 and U-2 OS cell lines were plated in 6-well plates and grown to $\sim 90 \%$ confluence. Twenty-four hours after transfection, the cells were serum starved for $6 \mathrm{~h}$. Then, a streak was created across the well using a $200 \mu \mathrm{L}$ pipette tip. The cells were washed with PBS for three times, and medium without serum was added into the wells. The progression of migration was photographed immediately and again at $24 \mathrm{~h}$ after wounding. The migration distance was measured randomly at five sites perpendicular to the wound to estimate the migration ability. Each experiment was replicated three times.

\section{Cell invasion assay}

Cell invasive ability was evaluated using 24-well transwell plates (Costar; Corning Incorporated, Corning, NY, USA). Before cell seeding, the matrigel (BD Biosciences, San Jose, CA, USA) was diluted in serum-free medium at a concentration of $1 \mathrm{mg} / \mathrm{mL}$. Hundred microliters of diluted matrigel per well was added into the top chamber and incubated for $4 \mathrm{~h}$ at $37^{\circ} \mathrm{C}$. Twenty-four hours after transfection, $1 \times 10^{4}$ cells in $200 \mu \mathrm{L}$ of serum-free medium were placed on the top chamber. The low chamber was filled with $500 \mu \mathrm{L}$ of DMEM supplemented with 20\% FBS (Invitrogen). After $24 \mathrm{~h}$ of incubation, cells invading into the bottom of the inserts were fixed with methanol and stained with crystal violet. Then, the invaded cells were observed and quantified by counting in five random high-magnification fields using microscopy. Each experiment was replicated three times.

\section{Statistical analysis}

All data management and statistical analyses were carried out using the SPSS software version 17.0 (SPSS Inc., Chicago, IL, USA). Data are shown as mean $\pm \mathrm{SD}$. The relationship between the CEACAM6 expression and the clinicopathologic features of osteosarcoma was evaluated using the chi-square and Fisher's exact tests. Survival curves were analyzed using the Kaplan-Meier method and the log-rank test. Other comparisons were estimated using unpaired two-sided Student's $t$-test. $P<0.05$ was considered statistically significant in all cases.

\section{Results \\ CEACAM6 is overexpressed}

\section{in metastatic osteosarcoma}

To explore the clinical significance and biological role of CEACAM6 in osteosarcoma, we first analyzed its expression in osteosarcoma cells and tissues. As shown in Figure 1A and $\mathrm{B}$, the mRNA and protein levels of CEACAM6 were expressed at high levels in osteosarcoma cells (MG-63, HOS, U-2 OS, Saos-2 and 143B) compared with FOB or NIH3T3 cells. Notably, CEACAM6 mRNA and protein levels were significantly higher in tumors with metastasis than those in tumors without metastasis (Figure 1C and D). Similar result was obtained when analyzing the mRNA expression of CEACAM6 in osteosarcoma using publicly published dataset GSE9508 and GSE14359 (Figure 1E and F). Moreover, the expression of CEACAM6 was further assessed by IHC staining in 91 paraffin-embedded osteosarcoma tissues (Figure 2A). Statistical analysis confirmed that CEACAM6 was significantly upregulated in metastatic osteosarcoma.

\section{CEACAM6 expression is associated with the clinicopathologic characteristics of osteosarcoma}

We next assessed the clinical relevance of CEACAM6 in osteosarcoma. Ninety-one osteosarcoma patients, whose characteristics are listed in Table 1, were successfully followed up. Correlation analysis showed that high expression of CEACAM6 was significantly associated with lung metastasis status $(P=0.006)$ in patients with osteosarcoma (Table 2). Moreover, Kaplan-Meier survival analyses showed that osteosarcoma patients with high CEACAM6 protein expression had a significantly shorter OS time (Figure 2B) and lung metastasis-free survival (LMFS) time (Figure 2C) than those with low CEACAM6 expression. Taken together, these data suggest that high CEACAM6 protein expression might contribute to osteosarcoma metastasis, leading to poor clinical outcome.

\section{CEACAM6 knockdown attenuates osteosarcoma cell migration and invasion in vitro}

To further investigate the potential biological functions of CEACAM6 in osteosarcoma cells, we performed migration 
A

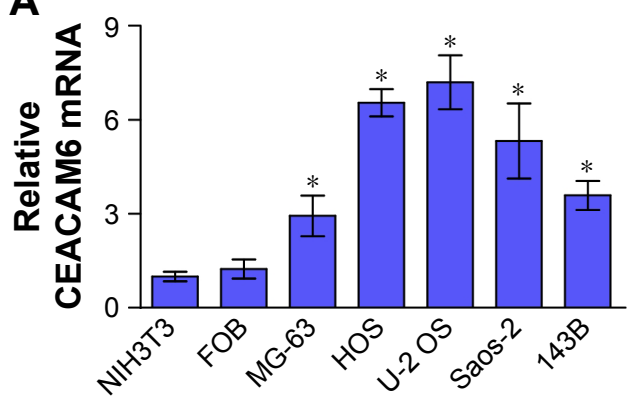

C

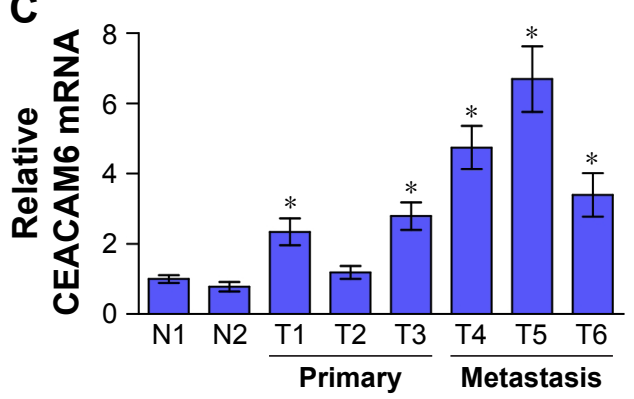

B

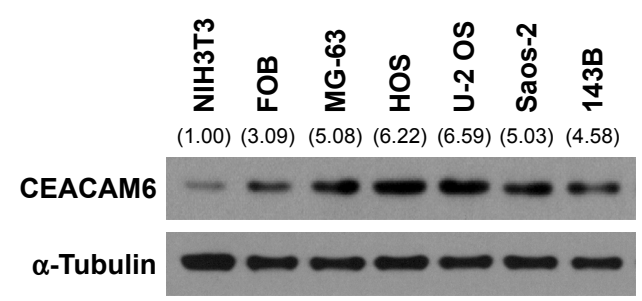

D

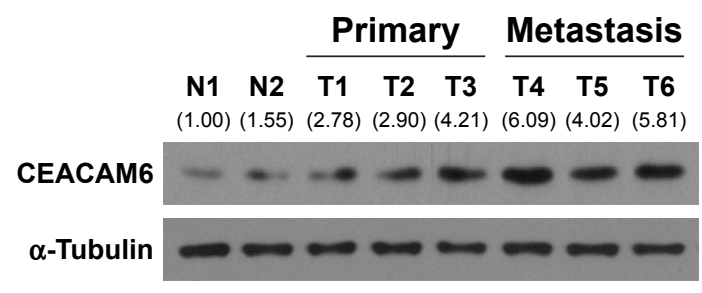

GSE14359

211657_at
203757 s at
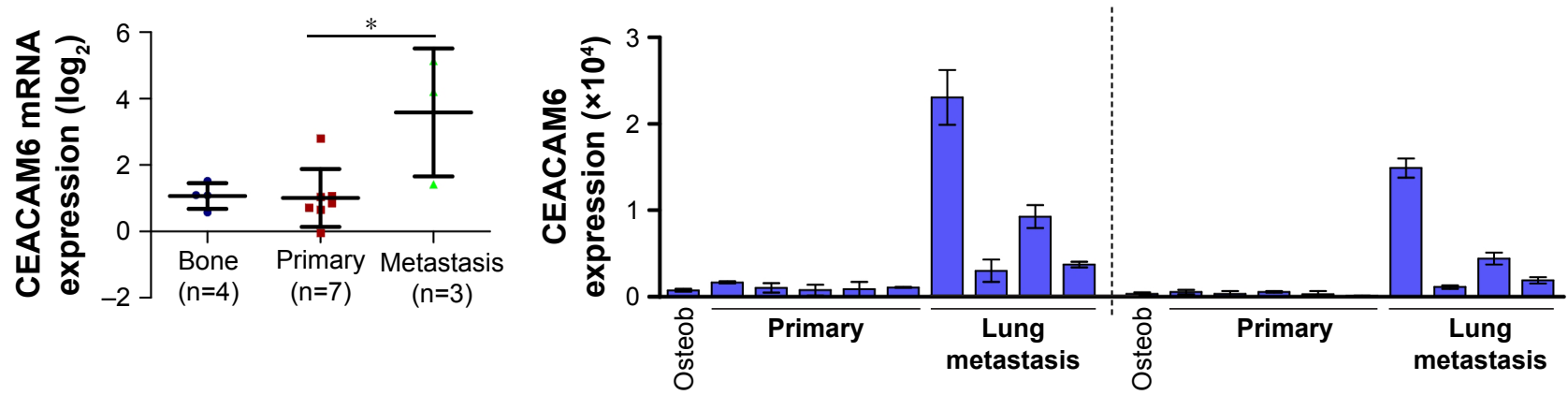

Figure I CEACAM6 is overexpressed in metastatic osteosarcoma.

Notes: Real-time PCR $(\mathbf{A})$ and Western blot $(\mathbf{B})$ analysis of CEACAM6 expression in human osteosarcoma cell lines. Real-time PCR (C) and Western blot (D) analysis of CEACAM6 expression in human osteosarcoma tissues. ( $N, n=2$; primary, $n=3$; metastasis, $n=3)$. CEACAM6 mRNA expression levels were significantly upregulated in metastatic osteosarcoma compared with non-metastatic osteosarcoma samples by analyzing GSE9508 (E) or GSEI4359 (F) dataset of osteosarcoma. $\alpha$-Tubulin was used as a protein loading control, and the integrated densities of CEACAM6 were quantified and normalized to $\alpha$-tubulin using ImageJ software. mRNA expression was normalized to GAPDH. Error bars represent the mean $\pm S D$ of three independent experiments. $* P<0.05$. Primary denotes non-metastatic osteosarcoma; metastasis denotes metastatic osteosarcoma. Abbreviations: CEACAM6, carcinoembryonic antigen-related cell adhesion molecule 6; N, adjacent non-tumor tissues; OS, overall survival; Osteob, non-neoplastic primary osteoblast cells; PCR, polymerase chain reaction.

and invasion assays. MG-63 and U-2 OS cells were transduced with lentiviruses carrying either CEACAM6-siRNA or control siRNA (Figure 3A and B). As shown in Figure 3C, wound-healing assays indicated that silencing CEACAM6 significantly decreased the cell migration distance of MG-63 and U-2 OS osteosarcoma cell lines. Matrigel-coated transwell experiments revealed that CEACAM6 knockdown reduced the number of invading cells in MG-63 and U-2 OS cells (Figure 3D). Taken together, these results suggest that CEACAM6 knockdown attenuates the metastatic ability of osteosarcoma cells.

\section{CEACAM6 facilitates EMT in osteosarcoma cell lines}

EMT is known to be associated with the metastatic ability of osteosarcoma cells. ${ }^{19}$ To investigate the mechanism of CEACAM6-mediated promotion in the invasiveness and migration of osteosarcoma cells, we next sought to identify whether CEACAM6 alters the expression of EMT markers. Notably, knocked down of CEACAM6 produced a dramatic shift in morphology, from a stick-like or long spindle-shaped mesenchymal-like cell population to a short spindle-shaped or round and flat epithelial-like cell 


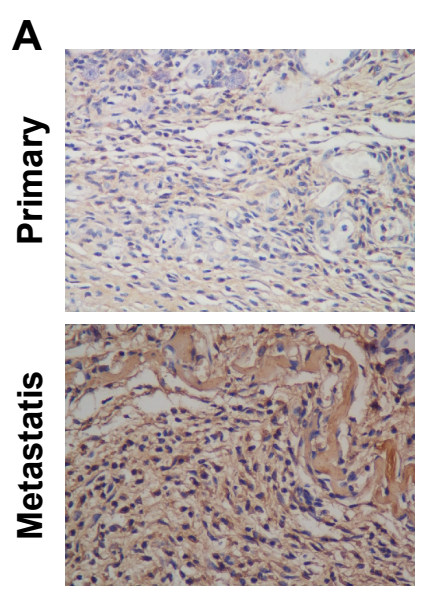

B

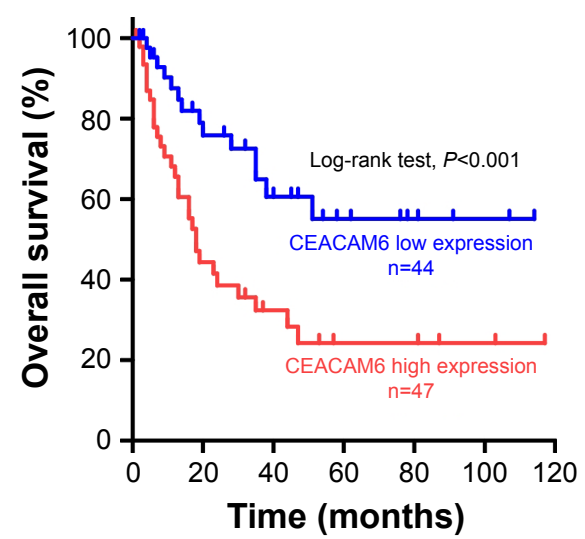

C

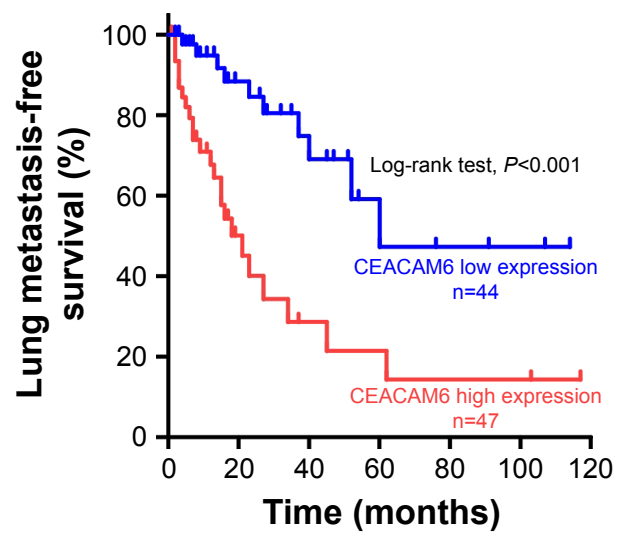

Figure 2 CEACAM6 expression is associated with the clinicopathologic characteristics of osteosarcoma.

Notes: (A) Representative immunohistochemical analysis of CEACAM6 expression in primary and metastatic osteosarcoma tissues. Kaplan-Meier analysis of overall survival (B) and lung metastasis-free survival (C) curves for osteosarcoma patients with low CEACAM6 expression ( $\mathrm{n}=44$ ) versus high CEACAM6 expression ( $\mathrm{n}=47$; log-rank test). Primary denotes non-metastatic osteosarcoma; metastasis denotes metastatic osteosarcoma.

Abbreviation: CEACAM6, carcinoembryonic antigen-related cell adhesion molecule 6.

population (Figure 4A). Moreover, as shown in Figure 4B, we found that the expression levels of $\mathrm{N}$-cadherin, vimentin and fibronectin were decreased, whereas E-cadherin and $\gamma$-catenin expression levels were increased, when CEACAM6

Table I Clinicopathologic characteristics of 91 osteosarcoma patients

\begin{tabular}{|c|c|}
\hline Characteristics & $\begin{array}{l}\text { Number of } \\
\text { cases (\%) }\end{array}$ \\
\hline \multicolumn{2}{|l|}{ Gender } \\
\hline Female & $33(36.3)$ \\
\hline Male & $58(63.7)$ \\
\hline \multicolumn{2}{|l|}{ Age (years) } \\
\hline$\leq 20$ & $65(71.4)$ \\
\hline $21-40$ & $24(26.4)$ \\
\hline$>40$ & $2(2.2)$ \\
\hline \multicolumn{2}{|l|}{ Location } \\
\hline Distal femur & $48(52.7)$ \\
\hline Proximal tibia & $22(24.2)$ \\
\hline Proximal humerus & $10(11.0)$ \\
\hline Proximal femur & $6(6.6)$ \\
\hline Others & $5(5.5)$ \\
\hline \multicolumn{2}{|l|}{ Enneking } \\
\hline IIB & 70 (76.9) \\
\hline III & $21(23.1)$ \\
\hline \multicolumn{2}{|l|}{ Relapse } \\
\hline Yes & $8(8.8)$ \\
\hline No & 83 (9I.2) \\
\hline \multicolumn{2}{|l|}{ Lung metastasis } \\
\hline Yes & $34(37.4)$ \\
\hline No & $57(62.6)$ \\
\hline \multicolumn{2}{|l|}{ CEACAM6 expression } \\
\hline High & $47(5 \mid .6)$ \\
\hline Low & $44(48.4)$ \\
\hline \multicolumn{2}{|c|}{ Vital status (at follow-up) } \\
\hline Alive & $48(52.7)$ \\
\hline Dead & $43(47.3)$ \\
\hline
\end{tabular}

Abbreviation: CEACAM6, carcinoembryonic antigen-related cell adhesion molecule 6. was silenced in MG-63 and U-2 OS osteosarcoma cell lines. These results imply that CEACAM6 promotes osteosarcoma cell migration and invasion by regulating the EMT pathway.

Table 2 Correlation between the CEACAM6 expression and the clinicopathologic characteristics of osteosarcoma patients

\begin{tabular}{|c|c|c|c|}
\hline \multirow[t]{2}{*}{ Characteristics } & \multicolumn{2}{|c|}{$\begin{array}{l}\text { CEACAM6 } \\
\text { expression }\end{array}$} & \multirow[t]{2}{*}{$P$-values } \\
\hline & Low & High & \\
\hline Gender & & & 0.677 \\
\hline Female & 15 & 18 & \\
\hline Male & 29 & 29 & \\
\hline Age (years) & & & 0.110 \\
\hline$\leq 20$ & 31 & 34 & \\
\hline $21-40$ & II & 3 & \\
\hline$>40$ & $\mathrm{I}$ & I & \\
\hline Location & & & 0.078 \\
\hline Distal femur & 29 & 19 & \\
\hline Proximal tibia & 6 & 16 & \\
\hline Proximal humerus & 3 & 7 & \\
\hline Proximal femur & 3 & 3 & \\
\hline Others & 3 & 2 & \\
\hline Enneking & & & 0.939 \\
\hline IIB & 34 & 36 & \\
\hline III & 10 & II & \\
\hline Relapse & & & 0.520 \\
\hline Yes & 3 & 5 & \\
\hline No & $4 I$ & 42 & \\
\hline Lung metastasis & & & 0.006 \\
\hline Yes & 10 & 24 & \\
\hline No & 34 & 23 & \\
\hline Vital status (at follow-up) & & & 0.005 \\
\hline Alive & 30 & 18 & \\
\hline Dead & 14 & 29 & \\
\hline
\end{tabular}

Abbreviation: CEACAM6, carcinoembryonic antigen-related cell adhesion molecule 6. 


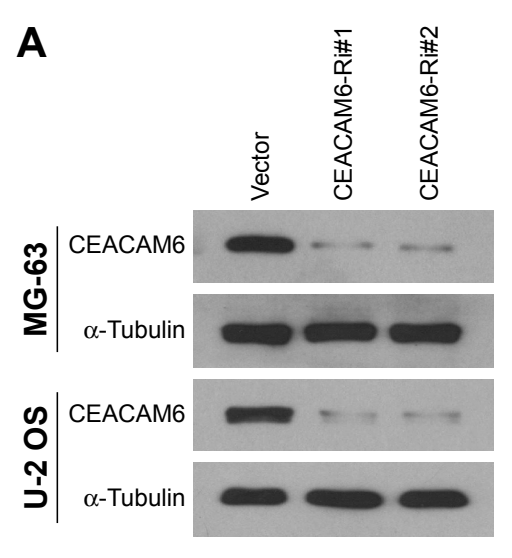
B
C
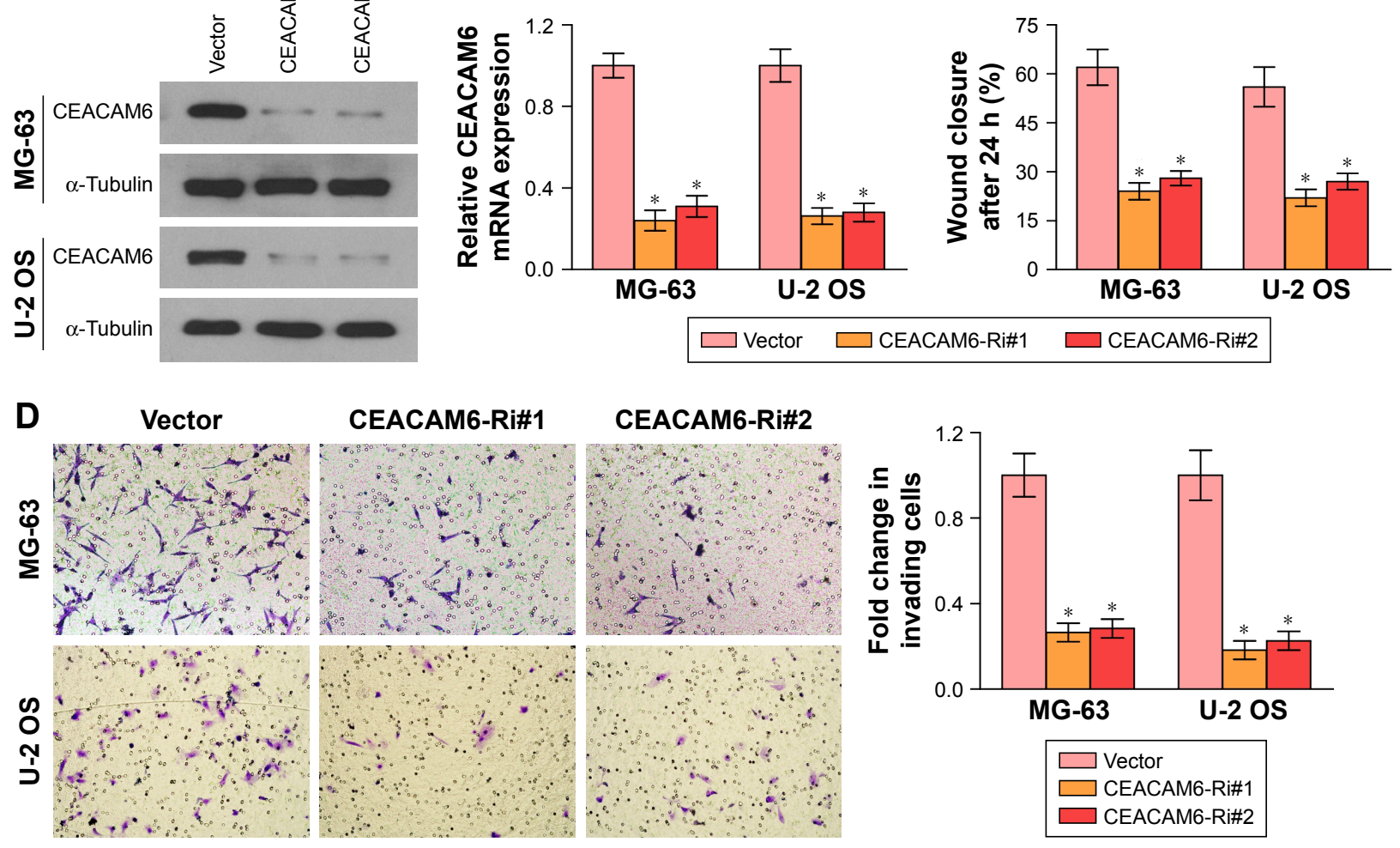

D
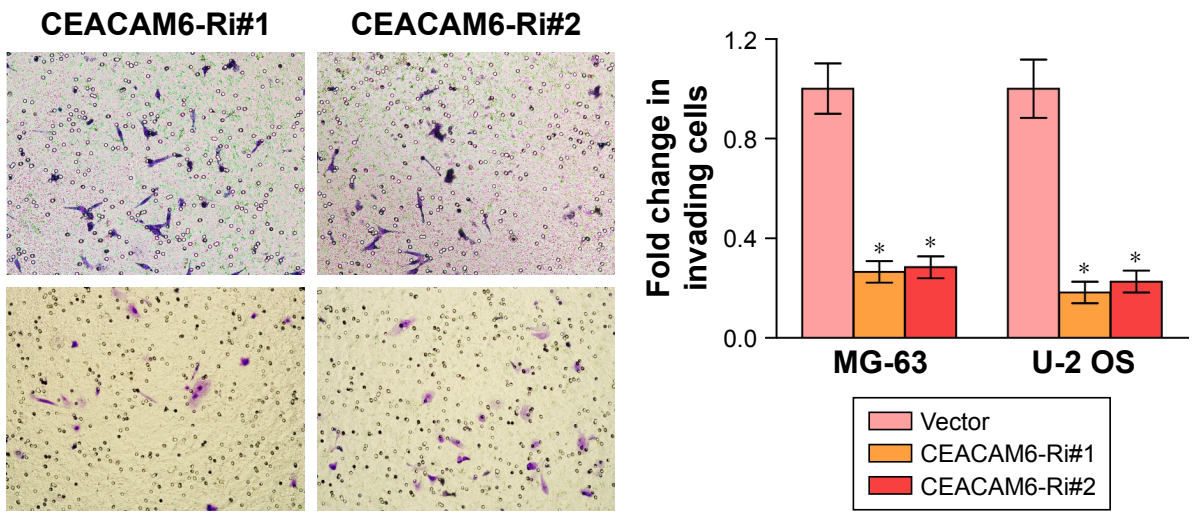

Figure 3 Knockdown of CEACAM6 inhibits osteosarcoma cell migration and invasion.

Notes: Western blot (A) and real-time PCR (B) analysis of CEACAM6 expression in the constructed MG-63 and U-2 OS cells. $\alpha$-Tubulin was used as a protein loading control, and mRNA expression was normalized to GAPDH. (C) Quantification of wound-healing assays for the indicated cell lines after $24 \mathrm{~h}$. (D) Representative micrographs (left panel) and quantification (right panel) of the invasiveness of indicated cells in the transwell matrix penetration assays. Data represent the mean \pm SD of three independent experiments; $* P<0.05$.

Abbreviations: CEACAM6, carcinoembryonic antigen-related cell adhesion molecule 6; OS, overall survival; PCR, polymerase chain reaction.

\section{Discussion}

The CEACAMs are a subgroup of the CEA family of immunoglobulin-related proteins, containing 12 different members. ${ }^{20}$ Previous studies have shown that CEACAMs are involved in morphogenesis, ${ }^{21}$ angiogenesis, ${ }^{22}$ cell proliferation, ${ }^{23}$ cell motility, ${ }^{24}$ apoptosis ${ }^{25}$ and regulation of cell matrix attachment. ${ }^{26}$ Dysregulation of CEACAMs contributes to human cancer malignancy, including osteosarcoma. ${ }^{8,15,27-30}$ CEACAM1 was elevated in osteosarcoma patients and positively correlated with tumor size, clinical stage, tumor grade and distant metastases..$^{30}$ The CEA assay could be used as an important tool to prognosticate the clinical recurrence of osteosarcoma during therapy. ${ }^{29}$ Similar to our results, recently, Liu et al found that CEACAM6 mRNA was notably highly expressed in metastatic osteosarcoma tissues when compared with the nonmetastatic osteosarcoma tissues..$^{15}$ However, its clinical significances, as well as biological functions, in osteosarcoma metastasis remain to be elucidated. Herein, we found that CEACAM6 was significantly associated with lung metastasis status $(P=0.006)$ in patients with osteosarcoma.
Moreover, Kaplan-Meier survival analyses showed that patients with high CEACAM6 expression had significantly worse OS and LMFS than those with low CEACAM6 expression, suggesting that CEACAM6 might be a promising prognostic biomarker for osteosarcoma.

Recently, more and more evidences revealed that CEACAM6 plays an important role in tumor metastasis. Kim et al discovered that high level of CEACAM6 is significantly correlated with colorectal cancer lymph nodal and distance metastasis, and promotes colorectal cancer cells invasion by regulating E-cadherin expression. ${ }^{11}$ Blumenthal et al demonstrated that targeting the $\mathrm{N}$ and A1B1 domains of CEACAM5/CEACAM6 using antibody Fabs obviously suppressed colorectal cancer cells lung metastasis. ${ }^{8}$ Moreover, CEACAM6 could promote tamoxifen-resistant breast cancer cells invasive properties, and might be an important biomarker of breast cancer metastasis..$^{31}$ In addition, CEACAM6 silencing impairs pancreatic adenocarcinoma cells anoikis resistance in vitro, and liver metastatic ability in vivo. ${ }^{32}$ Consistent with these results, our study confirmed 

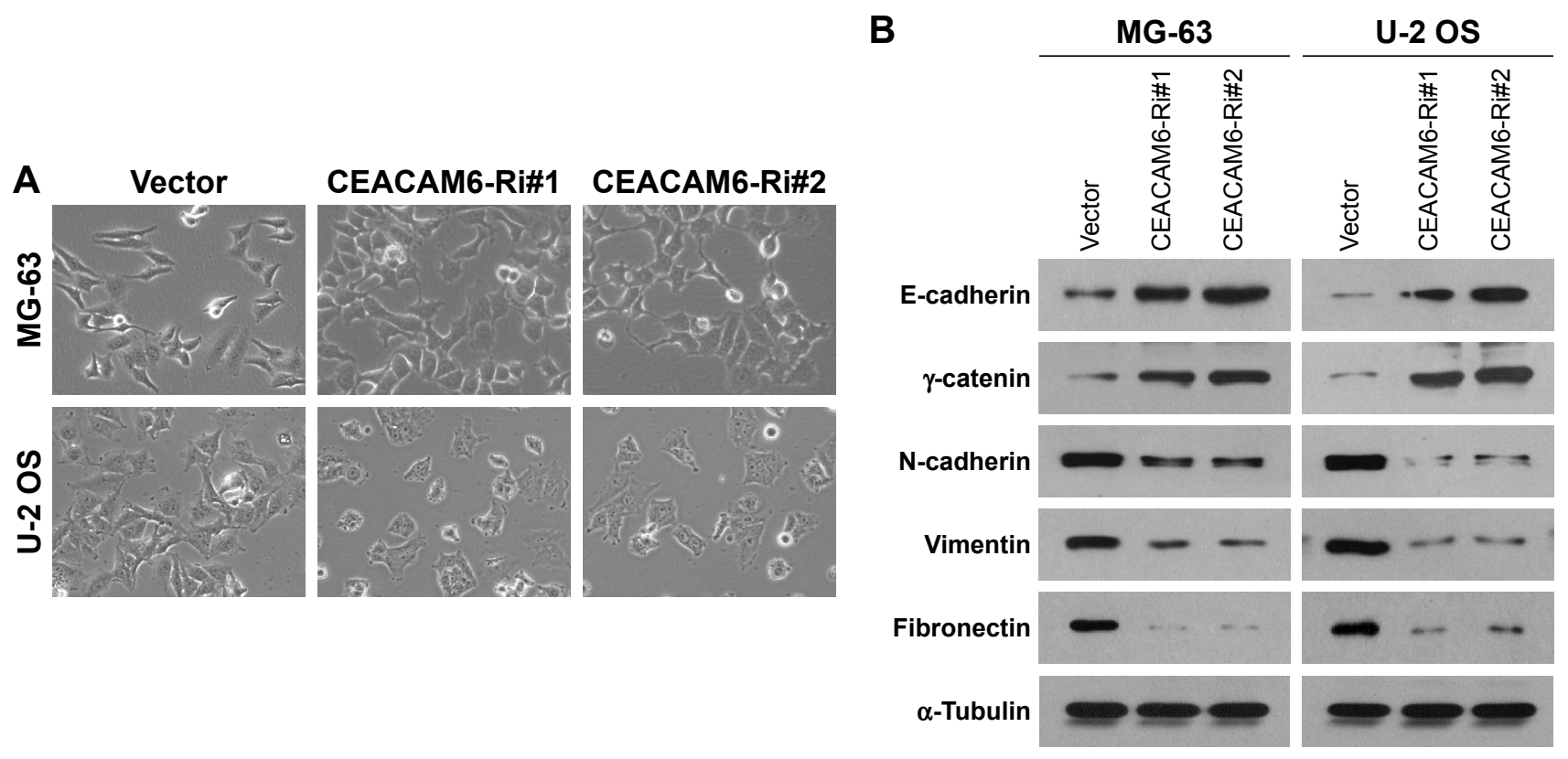

Figure 4 CEACAM6 facilitates EMT in osteosarcoma cell lines.

Notes: (A) Morphology change in the indicated cell lines. (B) Western blot analysis of E-cadherin, $\gamma$-catenin, $\mathrm{N}$-cadherin, vimentin and fibronectin expression in the constructed MG-63 and U-2 OS cells. $\alpha$-Tubulin was used as a protein loading control.

Abbreviations: CEACAM6, carcinoembryonic antigen-related cell adhesion molecule 6; EMT, epithelial-mesenchymal transition; OS, overall survival.

that CEACAM6 was upregulated in metastatic osteosarcoma. High CEACAM6 expression predicted quicker occurrence of lung metastasis event in osteosarcoma patients. Moreover, CEACAM6 gene silencing dramatically reduced osteosarcoma cells migration and invasion by regulating the EMT pathway. Taken together, these findings supported the crucial role of CEACAM6 in tumor metastasis, and it might be a useful therapeutic target against osteosarcoma metastasis.

EMT is a developmental program that guides proper cell movement during embryogenesis, ${ }^{33}$ and is commonly hijacked by cancer cells in the process of metastasis, conferring on epithelial cells properties that are critical to invasion and metastatic dissemination. ${ }^{34}$ The EMT program is characterized by downregulation of epithelial marker, such as E-cadherin, and upregulation of mesenchymal markers, such as $\mathrm{N}$-cadherin and vimentin. ${ }^{34}$ Herein, we found that E-cadherin and $\gamma$-catenin expression levels were increased, while $\mathrm{N}$-cadherin, vimentin and fibronectin expression levels were decreased in CEACAM6 silencing osteosarcoma cells, suggesting that CEACAM6 promotes osteosarcoma cells metastatic properties by regulating EMT pathway. However, the molecular mechanism remains to be further investigated.

\section{Conclusion}

Our study revealed that CEACAM6 is upregulated in metastatic osteosarcoma tissues and promotes osteosarcoma cells migration and invasion by regulating the EMT pathway.
CEACAM6 may serve as a predictive biomarker for poor prognosis and lung metastasis in osteosarcoma. Targeting CEACAM6 might also represent a potentially novel therapeutic strategy for osteosarcoma.

\section{Author contributions}

ZW was responsible for most of the experiments, data collection and analysis. CL and HW conducted the molecular cloning, data analysis and reviewing. CL and XY performed the immunohistochemical staining. ZM and WL supervised the project. ZW wrote the manuscript. ZM and WL designed and conceived the study. All authors contributed toward data analysis, drafting and revising the paper and agree to be accountable for all aspects of the work. All authors read and approved the manuscript.

\section{Disclosure}

The authors report no conflicts of interest in this work.

\section{References}

1. Mirabello L, Troisi RJ, Savage SA. Osteosarcoma incidence and survival rates from 1973 to 2004: data from the Surveillance, Epidemiology, and End Results Program. Cancer. 2009;115(7):1531-1543.

2. Kempf-Bielack B, Bielack SS, Jürgens H, et al. Osteosarcoma relapse after combined modality therapy: an analysis of unselected patients in the cooperative Osteosarcoma Study Group (COSS). J Clin Oncol. 2005;23(3):559-568.

3. Goorin AM, Schwartzentruber DJ, Devidas M, et al. Presurgical chemotherapy compared with immediate surgery and adjuvant chemotherapy for nonmetastatic osteosarcoma: Pediatric Oncology Group Study POG-8651. J Clin Oncol. 2003;21(8):1574-1580. 
4. Meyers PA, Heller G, Healey J, et al. Chemotherapy for nonmetastatic osteogenic sarcoma: the Memorial Sloan-Kettering experience. J Clin Oncol. 1992;10(1):5-15.

5. Marko TA, Diessner BJ, Spector LG. Prevalence of metastasis at diagnosis of osteosarcoma: an international comparison. Pediatr Blood Cancer. 2016;63(6):1006-1011.

6. Duchman KR, Gao Y, Miller BJ. Prognostic factors for survival in patients with high-grade osteosarcoma using the Surveillance, Epidemiology, and End Results (SEER) Program database. Cancer Epidemiol. 2015;39(4):593-599.

7. Paxton RJ, Mooser G, Pande H, Lee TD, Shively JE. Sequence analysis of carcinoembryonic antigen: identification of glycosylation sites and homology with the immunoglobulin supergene family. Proc Natl Acad Sci US A. 1987;84(4):920-924.

8. Blumenthal RD, Hansen HJ, Goldenberg DM. Inhibition of adhesion, invasion, and metastasis by antibodies targeting CEACAM6 (NCA-90) and CEACAM5 (Carcinoembryonic Antigen). Cancer Res. 2005;65(19):8809-8817.

9. Cameron S, de Long LM, Hazar-Rethinam M, et al. Focal overexpression of CEACAM6 contributes to enhanced tumourigenesis in head and neck cancer via suppression of apoptosis. Mol Cancer. 2012;11:74.

10. Chen J, Li Q, An Y, et al. CEACAM6 induces epithelial-mesenchymal transition and mediates invasion and metastasis in pancreatic cancer. Int J Oncol. 2013;43(3):877-885.

11. Kim KS, Kim JT, Lee SJ, et al. Overexpression and clinical significance of carcinoembryonic antigen-related cell adhesion molecule 6 in colorectal cancer. Clin Chim Acta. 2013;415:12-19.

12. Tsang JY, Kwok YK, Chan KW, et al. Expression and clinical significance of carcinoembryonic antigen-related cell adhesion molecule 6 in breast cancers. Breast Cancer Res Treat. 2013;142(2):311-322.

13. Litkouhi B, Litkouhi B, Fleming E, et al. Overexpression of CEACAM6 in borderline and invasive mucinous ovarian neoplasms. Gynecol Oncol. 2008;109(2):234-239.

14. Ieta K, Tanaka F, Utsunomiya T, Kuwano H, Mori M. CEACAM6 gene expression in intrahepatic cholangiocarcinoma. Br J Cancer. 2006; 95(4):532-540.

15. Liu K, He Q, Liao G, Han J. Identification of critical genes and gene interaction networks that mediate osteosarcoma metastasis to the lungs. Exp Ther Med. 2015;10(5):1796-1806.

16. Li Q, Ye L, Guo W, Wang M, Huang S, Peng X. PHF21B overexpression promotes cancer stem cell-like traits in prostate cancer cells by activating the Wnt/beta-catenin signaling pathway. J Exp Clin Cancer Res. 2017;36(1):85.

17. Li Q, Ye L, Zhang X, et al. FZD8, a target of p53, promotes bone metastasis in prostate cancer by activating canonical Wnt/beta-catenin signaling. Cancer Lett. 2017;402:166-176.

18. Li Q, Ye L, Guo W, Wang M, Huang S, Peng X. Overexpression of TACC3 is correlated with tumor aggressiveness and poor prognosis in prostate cancer. Biochem Biophys Res Commun. 2017;486(4):872-878.
19. Niinaka Y, Harada K, Fujimuro M, et al. Silencing of autocrine motility factor induces mesenchymal-to-epithelial transition and suppression of osteosarcoma pulmonary metastasis. Cancer Res. 2010;70(22): 9483-9493.

20. Kuespert K, Pils S, Hauck CR. CEACAMs: their role in physiology and pathophysiology. Curr Opin Cell Biol. 2006;18(5):565-571.

21. Yokoyama S, Chen CJ, Nguyen T, Shively JE. Role of CEACAM1 isoforms in an in vivo model of mammary morphogenesis: mutational analysis of the cytoplasmic domain of CEACAM1-4S reveals key residues involved in lumen formation. Oncogene. 2007;26(55):7637-7646.

22. Horst AK, Ito WD, Dabelstein J, et al. Carcinoembryonic antigen-related cell adhesion molecule 1 modulates vascular remodeling in vitro and in vivo. J Clin Invest. 2006;116(6):1596-1605.

23. Singer BB, Scheffrahn I, Kammerer R, Suttorp N, Ergun S, Slevogt H. Deregulation of the CEACAM expression pattern causes undifferentiated cell growth in human lung adenocarcinoma cells. PLoS One. 2010;5(1):e8747.

24. Klaile E, Muller MM, Kannicht C, Singer BB, Lucka L. CEACAM1 functionally interacts with filamin $\mathrm{A}$ and exerts a dual role in the regulation of cell migration. J Cell Sci. 2005;118(Pt 23):5513-5524.

25. Singer BB, Klaile E, Scheffrahn I, et al. CEACAM1 (CD66a) mediates delay of spontaneous and Fas ligand-induced apoptosis in granulocytes. Eur J Immunol. 2005;35(6):1949-1959.

26. Muenzner P, Rohde M, Kneitz S, Hauck CR. CEACAM engagement by human pathogens enhances cell adhesion and counteracts bacteriainduced detachment of epithelial cells. J Cell Biol. 2005;170(5): 825-836.

27. Jantscheff $P$, Terracciano L, Lowy A, et al. Expression of CEACAM6 in resectable colorectal cancer: a factor of independent prognostic significance. J Clin Oncol. 2003;21(19):3638-3646.

28. Hong KP, Shin MH, Yoon S, et al. Therapeutic effect of anti CEACAM6 monoclonal antibody against lung adenocarcinoma by enhancing anoikis sensitivity. Biomaterials. 2015;67:32-41.

29. Cortes EP, Chu TM, Wang JJ, Holyoke D, Wallace HJ, Murphy GP. Carcinoembryonic antigen in osteosarcoma. J Surg Oncol. 1977;9(3): 257-265.

30. Yu H, Yu J, Ren Y, Yang Y, Xiao X. Serum CEACAM1 level is associated with diagnosis and prognosis in patients with osteosarcoma. PLoS One. 2016;11(4):e0153601.

31. Lewis-Wambi JS, Cunliffe HE, Kim HR, Willis AL, Jordan VC. Overexpression of CEACAM6 promotes migration and invasion of oestrogendeprived breast cancer cells. Eur J Cancer. 2008;44(12):1770-1779.

32. Duxbury MS, Ito H, Zinner MJ, Ashley SW, Whang EE. CEACAM6 gene silencing impairs anoikis resistance and in vivo metastatic ability of pancreatic adenocarcinoma cells. Oncogene. 2004;23(2):465-473.

33. Hay ED. An overview of epithelio-mesenchymal transformation. Acta Anat (Basel). 1995;154(1):8-20.

34. Kalluri R, Weinberg RA. The basics of epithelial-mesenchymal transition. J Clin Invest. 2009;119(6):1420-1428.
OncoTargets and Therapy

\section{Publish your work in this journal}

OncoTargets and Therapy is an international, peer-reviewed, open access journal focusing on the pathological basis of all cancers, potential targets for therapy and treatment protocols employed to improve the management of cancer patients. The journal also focuses on the impact of management programs and new therapeutic agents and protocols on
Dovepress

patient perspectives such as quality of life, adherence and satisfaction The manuscript management system is completely online and includes a very quick and fair peer-review system, which is all easy to use. Visit http://www.dovepress.com/testimonials.php to read real quotes from published authors. 